\title{
Mathematical modeling of separation of watered oil-containing mixture
}

\author{
V.A. Zelenskiy ${ }^{1}$, A.A. Sushin ${ }^{1}$, A.I. Shchodro ${ }^{1}$ \\ ${ }^{1}$ Samara National Research University, 34 Moskovskoe Shosse, 443086, Samara, Russia
}

\begin{abstract}
A mathematical description of the separation process of the watered oil-containing mixture has been proposed. The mathematical model is based on the determination of the speed of movement of oil globules in a constrained flow. The formula for determining the velocity of the globules is derived from theoretical provisions and experimental data. The key issue of simulation is determining the separation time. This characteristic is calculated as a time period from the moment of arrival of the portion of oil-containing mixture to the first chamber of the separator to the moment of formation of the continuous phase layer of the given thickness on the surface. The mathematical model enables us to determine the separation time taking into account geometric characteristics of the separation device at the high level of water content. Thus, a topical problem of improving the performance of the separation device without sacrificing the quality of the commercial oil is solved.
\end{abstract}

Keywords: separation device; watered oil-containing mixture; oil globules; constrained flow; separation time

\section{Introduction}

The separation of the oil-containing mixture is one of the main technological processes in the oil treatment control system (OTCS). In order for the OTCS to function effectively, it is necessary to determine the optimal separation time. The duration of the separation process is directly related to the performance improvement of the separation device and the OTCS as a whole without sacrificing the quality of the commercial oil [1]. Furthermore, the separation time is the most important parameter for managing the process in automated mode [2]. As a rule, the requirements for performance and oil quality are mutually contradictory. Curing mixture in separators (clarifying tanks) reduces OTCS efficiency. On the other hand, the quality of the commercial oil should be monitored when reducing the time of separation. One of the most important indicators of oil quality is its residual water content. The measurement of water content in oil is performed according to GOST P 51858-2002. The thirdgroup oil standard is under $1 \%$. More strict requirements are applied to oil of the 1 st and 2 nd group. The residual water content of these groups shall not exceed $0.5 \%$. The percentage of water content in the oil-containing mixture will be called watercut. The oil and gas industry in many regions (Tatarstan, Bashkortostan, Samara, Orenburg regions) operates wells with watercut varying from $70 \%$ to $90 \%$. The profitability of such wells can be achieved only through equipment upgrade, employment of upto-date technologies and IT. This should be preceded by the analysis of processes taking place in OTCS based on their mathematical models. As a result, the simulation of a separation process is a relevant scientific task.

\section{Physical description of a separation process}

The separation process of the oil-containing mixture takes place in a separation device, also called an "oil and gas separator" or simply a "separator" [2]. The fragment of separator (first chamber) is shown in Figure 1. The main elements are: gas pressure sensor (1), fluid-level sensor (2), partition wall between separator's chambers of $h_{\mathrm{p}}$ height (3), control device (4), temperature sensor (5), fluid pressure hydrostatic sensor (6), water-discharge valve (7), inlet valve for oil-containing mixture (8).

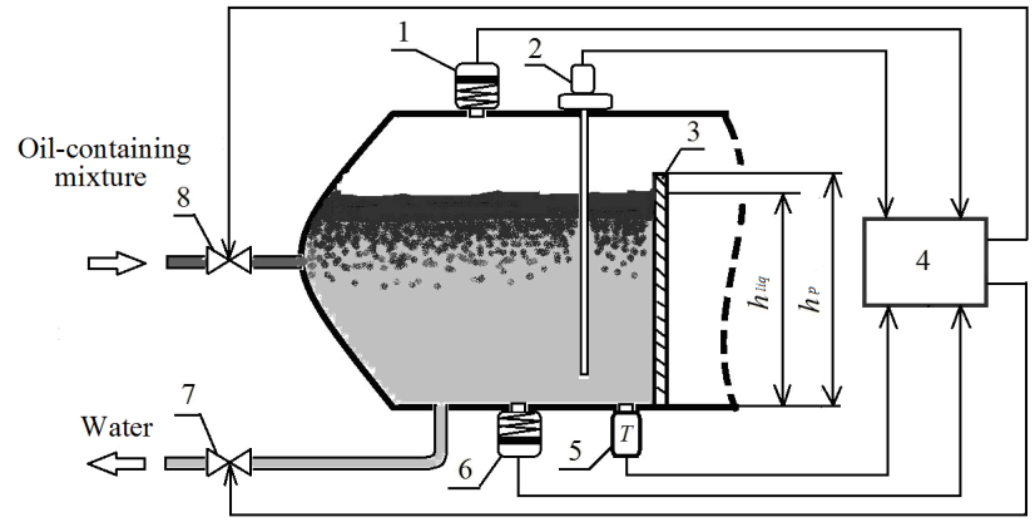

Fig. 1. The first chamber of separation device.

A complex mixture containing oil-associated gas, water, oil, metal salts and other impurities comes into the separator's chamber from an oil well through the inlet valve 8 . The velocity of separation of the gas phase is much higher than fluid demulsification, so the influence of the gas factor can be neglected. After the chamber is filled up to the height of $h_{l}$, the gravity clarifying of oil-containing mixture takes place. As a result, a lighter oil globule comes to the surface. Relatively heavy globules of water are depositing to the bottom of the chamber and through the valve 7 get into the water treatment device and further back to the well. The separation process begins with the destruction of the globule membranes, which leads to their adhesion. Then the globules become larger as a result of coagulation. Finally, coalescence of globules results in the formation of continuous phases of water and oil. Sensors 1, 2, 5, 6 readings are used to generate control signals in control device 4 . Thus, by 
indirect measuring of density the mixture watercut value is obtained [3]. This information is used to determine the separation time [4]. Three approaches to obtain this parameter can be highlighted.

1) Separation time is a period of time from the moment the first chamber of the separator is filled (up to a given level, not exceeding the overflow level) until full decomposition of the oil-containing mixture. This approach did not find practical use as it does not take into account the real velocity of demulsification process.

2) Separation time is a period of time from the moment of arrival of the portion of oil-containing mixture to the first chamber of oil and gas separator to the moment of its decomposition to the state determined by GOST P 51858-2002. This approach is more progressive than the first one, but does not take into account the continuous nature of OTCS operation.

3) Separation time is a period of time from the moment of arrival of the proportion of oil-containing mixture to the first chamber of the separator to the moment of formation of the continuous phase layer of the given thickness on the surface. This is the preferred method to be used in the framework of this research.

\section{Mathematical description of separation process}

There is no strict mathematical description of the stratification of the oil-containing mixture as Navier-Stokes equation is valid just for laminar flow and some special cases [5]. However, there are a large number of empirical and semi-empirical relationships that determine the nature of the processes in the stratification of emulsions that can be used as a basis [6, 7]. Under the conditions of strong watercut of oil mixture $(70 \% \ldots 90 \%)$, oil is a dispersed phase and water is a dispersed medium. The oil particle rising to the surface experiences the difference between the gravity force and the lifting Archimedes' force [7].

$$
\Delta F=\frac{\pi g}{6} d^{3} \Delta p
$$

where $\Delta \rho$ is the difference between dispersed phase and dispersed medium particles' density, $g$ is the gravitational acceleration, $d$ is the particle's diameter. Resistance force of the continuous medium:

$$
F_{c}=\xi_{o} \frac{\pi d^{2}}{4} \frac{\omega_{o}}{2} \rho_{c},
$$

where $\xi_{0}$ is the coefficient of hydraulic resistance of the continuous medium to the movement of a single particle in it, $\omega_{0}$ is the velocity of a single particle relative to the medium, $\rho_{\mathrm{c}}$ is the density of the continuous medium. Let us assume that the temperature at all points of a separation device chamber is the same. Then there is no thermal convection. If the particle's velocity in the medium is constant: $\Delta F=F_{c}$ the Reynolds criterion is determined by the following ratio:

$$
\operatorname{Re}_{o}=\frac{\omega_{0} d \rho_{c}}{\mu_{c}},
$$

where $\mu_{\mathrm{c}}$ is the dynamic viscosity of the continuous medium. Archimedes' criterion is as follows:

$$
A r=\frac{d^{3} g}{v_{c}^{2}} \frac{\rho_{\partial}-\rho_{c}}{\rho_{c}},
$$

where $v_{c}$ is the kinematic viscosity of continuous medium, $\rho_{d}$ is the density of the dispersed phase. Taking these criteria into account, we can derive the following equality:

$$
\xi_{o} \operatorname{Re}_{o}^{2}=\frac{4}{3} A r
$$

Under the conditions of constrained surfacing, which is characterized by the interaction between particles, the following equality is valid:

$$
\xi_{\partial} \operatorname{Re}_{\partial}{ }^{2}=\frac{4}{3} A r
$$

where $\xi_{d}$ is the coefficient of hydraulic resistance for the dispersed phase in the emulsion, $\operatorname{Re}_{\partial}$ is the Reynolds criterion under the conditions of constrained flow. Hence the following formula is obtained:

$$
\xi_{\partial} \operatorname{Re}_{\partial}^{2}=\xi_{o} \operatorname{Re}_{o}^{2} .
$$

Let us assume that

$$
\xi_{\partial}=f(\varphi) \xi_{o \partial}
$$

where $\xi_{\text {od }}$ is the coefficient of hydraulic resistance for a continuous medium for a single particle under the constrained flow conditions, $\varphi$ is the volume fraction of the dispersed phase within the system. It would be useful to define the type of function $f$ $(\varphi)$ for the small and large Reynolds criterion values. Experimental studies showed that the velocities of particles depositing are connected by the following relation:

$$
\omega_{o \partial}=\omega_{o}(1-\varphi)^{n},
$$

where $\omega_{0 d}$ is the depositing rate of the particle relative to the continuous medium in constrained flow conditions, $\omega_{0 d}$ is the rate of free depositing of the particle, and $n$ index is to be determined. Using the obtained parameter called the volume fraction of the dispersed phase, we have:

$$
\operatorname{Re}_{\partial}=(1-\varphi) \operatorname{Re}_{o}
$$


It is experimentally shown [7] that with small values of Reynolds criterion $\left(R_{e}<500\right)$ the hydraulic resistance coefficient of the medium equals:

$$
\zeta_{d}=\frac{24\left(1+0,15 \operatorname{Re}_{0}^{0.687}\right)}{0.843 \lg (\theta / 0.065) \operatorname{Re}}
$$

where $\theta$ is the coefficient of the particle shape, equal to the ratio of the surface area of the spherical particle to the surface area of the real particle of the same volume. With small values of Reynolds coefficient, we can assume that:

$$
f(\varphi) \approx(1-\varphi)^{-n}
$$

For large values of Reynolds coefficient Re the following expression is valid:

$$
f(\varphi) \approx(1-\varphi)^{-2 n} .
$$

It is experimentally shown [7] that the function $f(\varphi)$, both in case of large and small values of $\operatorname{Re}$ varies from $(1-\varphi)^{-4.65}$ to $(1-$ $\varphi)^{-4.78}$. Then we can take an average index and write:

$$
f(\varphi)=(1-\varphi)^{-4.72}
$$

Taking into account the expressions derived, it is obtained that the ratio of the particle depositing rate relative to the continuous medium under the constrained flow conditions to the particle's free depositing rate is equal to:

$$
\omega_{o d} / \omega_{0}=(1-\varphi)^{-4.72} \text {. }
$$

There exist empirical formulas which enable us to account for the influence of constrainity [7]. For example, when $\varphi<0.3$, the following formula is applied:

For $\varphi>0,3$ the following formula is applied:

$$
\omega_{o d} / \omega_{o}=(1-\varphi)^{2} 10^{-1.82 \varphi}
$$

\begin{tabular}{|c|c|c|c|}
\hline \multirow[t]{2}{*}{ Watercut, \% } & \multicolumn{3}{|c|}{ Results of calculations according to formulas, relative units } \\
\hline & $\omega_{o d} / \omega_{0}=(1-\varphi)^{-4.72}$ & $\omega_{o d} / \omega_{o}=(1-\varphi)^{2} 10^{-1.82 \varphi}$ & $\omega_{o d} / \omega_{o}=\frac{0.123}{\varphi}(1-\varphi)^{3}$ \\
\hline 5 & 0.7558 & 0.7319 & \\
\hline 10 & 0.6095 & 0.5327 & \\
\hline 20 & 0.3504 & 0.2768 & \\
\hline 30 & 0.1871 & & 0.1406 \\
\hline 40 & 0.0906 & & 0.0664 \\
\hline 50 & 0.0385 & & 0.0308 \\
\hline 60 & 0.0135 & & 0.0131 \\
\hline 70 & 0.0035 & & 0.0047 \\
\hline
\end{tabular}

$$
\omega_{o d} / \omega_{o}=\frac{0.123}{\varphi}(1-\varphi)^{3} .
$$

Table 1 shows comparative data of the calculation of the velocity of the oil globules rising for the known relations and calculated according to the obtained formula. Numerical values are reduced to the velocity of oil globules freely rising to the surface, i.e. relative ones.

Data for the watercut degree from $70 \%$ to $90 \%$ have not been found. The velocities of oil globules rising distributed by fractions are of practical interest. The known relations are obtained for a case when the dispersed phase is water. In our case, the dispersed phase is oil. In paper [7] it is assumed that the distribution of water drops in oil after filling the chamber of separator is uniform. Therefore, the watercut $B$ in any vertical section is the same. The relative velocity of the constrained surfacing of oil globules of $d_{i}$ diameter in this case equals:

$$
\left(w_{0 d} / w_{0}\right)_{i}=\left[\frac{1-B}{1-B \sqrt{1-\left(d_{i} / d_{\max }\right)^{2}}}\right]^{4.72},
$$

where $d_{\max }$ is the maximum size of the globule. Generally, it is suggested to define the separation time through the velocity of the oil globules rising and the geometric parameters of the oil and gas separator. The velocity of constrained rising of oil globules is as follows:

$$
w_{0}=\frac{\left(p_{d}-p_{c}\right) d^{2} g}{18 \mu_{c}},
$$


where $p_{d}, p_{c}$ is the density of dispersed and continuous medium, $\mathrm{kg} / \mathrm{m} 3 ; \mu_{c}$ is the viscosity of the continuous medium, Pa $\mathrm{s} ; d$ is the diameter of the globule; $g$ is the gravitational acceleration, $\mathrm{m} / \mathrm{s}^{2}$. While rising, globules of different sizes are moving at different speeds. It is proposed to use the following expression to describe the calculation of the constrained rising of globules:

$$
\left(w_{0 d} / w_{0}\right)_{i}=\frac{\left(p_{d}-p_{c}\right) d^{2} g}{18 \mu_{c}}\left[\frac{1-B}{1-B \sqrt{1-\left(d_{i} / d_{\max }\right)^{2}}}\right]^{4.72},
$$

The equation obtained enables us to calculate the spectrum of velocities of constrained rising of the oil globules, taking into account the change of watercut of emulsion according to the height of the partition wall between the chambers of the separation device. In Table 2 there are given the data for the watercut values of the studied range from $70 \%$ to $90 \%$. In accordance with table 2 and geometric characteristics of the separation device, it is possible to determine the separation time for oil-containing mixture, taking into account the composition of the mixture and watercut value.

$$
\text { Table 2. The velocity of oil globules rising in the chamber of separation device. }
$$

\begin{tabular}{|c|c|c|c|c|c|c|}
\hline Globules & \multicolumn{6}{|c|}{ The velocity of the oil globules rising, $\mathrm{cm} / \mathrm{s}$} \\
\hline 50 & 78.2609 & 128.35 & 184.70 & 240.26 & 329.47 & 417.91 \\
\hline 60 & 54.5455 & 89.45 & 128.76 & 167.50 & 229.70 & 291.35 \\
\hline 80 & 30.7692 & 50.46 & 72.62 & 94.46 & 129.54 & 164.31 \\
\hline 100 & 19.6721 & 32.26 & 46.42 & 60.39 & 82.81 & 105.04 \\
\hline 150 & 9.6257 & 15.79 & 22.73 & 29.56 & 40.54 & 51.42 \\
\hline 200 & 4.9113 & 8.05 & 11.59 & 15.07 & 20.67 & 26.22 \\
\hline
\end{tabular}

$\begin{array}{llllll}\text { Watercut, } \% & 90 & 85 & 80 & 75 & 70\end{array}$

\section{Conclusion}

The obtained relations enable us to mathematically describe the separation process through the velocity of the globules in the case of a high watercut value for oil-containing mixture, which demonstrates the scientific novelty. Using the mathematical model data and knowing the geometric characteristics of the separation device, separation time can be calculated. The precise definition of separation time improves the performance of the separation device without sacrificing the quality of the commercial oil, which is of great practical importance.

\section{Acknowledgements}

The authors express their gratitude for LLC Coordination (Ufa), LLC "GIRS", "Neftestroy" Educational Centre (Samara) for the fruitful cooperation and experimental data provided.

\section{References}

[1] Zelenskiy VA, Shchodro AI. Increasing the efficiency of separation by controlling the differential density of the oil and gas mixture. Bulletin of Samara State Technical University. Series "Technical Sciences" 2015; 1(45): 178-183.

[2] Zelenskiy VA, Shchodro AI. The automated control system of the oil and gas separator providing control over the density of the oil-containing mixture. Bulletin of Samara State Technical University. Series "Technical Sciences" 2016; 1(49): 15-23.

[3] Zelenskiy VA, Shchodro AI. Analysis of errors of measuring the density of the oil-containing mixture and their impact on the determination of the separation time. Bulletin of Samara Scientific Centre of the Russian Academy Sciences 2016; 18(3): 896-901.

[4] Zelenskiy VA, Shchodro AI. Method, mathematical model, and the algorithm of control over the oil separation process. Bulletin of Samara State Technical University. Series "Technical Sciences" 2016; 3(51): 21-28.

[5] Cochin NE, Kibel IA, Rose NV. Theoretical hydromechanics, part 2. Moscow: Fizmatlit, 1963; 727 p.

[6] Ponomarev SV, Mishchenko SV. Methods and devices for measuring the effective thermal performance of the flows of technological liquids. Tambov: Publ. Tambov State Technical University, 1997; 249 p.

[7] Astarita J, Maruchchi J. The basics of hydromechanics of non-Newtonian fluids. Moscow: "Mir", 1978; 312 p. 